\title{
Containment of Cattle Brucellosis Outbreaks With a Small Dose of Vaccine From Strain 82
}

\section{Alexei Novitsky, Valentina Pleshakova, Nadezhda Lescheva, and Vasily Vlasenko}

Omsk State Agrarian University, 644008, Institutskaya ploshchad str., 1, Omsk, Russia

ORCID:

Alexei Novitsky: http://orcid.org/0000-0001-8351-2818

\section{Abstract}

Improving the system of anti-brucellosis measures is the ultimate goal of all studies devoted to the problem of brucellosis. Cattle brucellosis outbreaks in the brucellosisfree regions in the Russian Federation indicate a premature cessation of immunization procedures and the need for their continuation. Meanwhile, there is an urgent problem of developing anti-brucellosis immunity in the adult cattle: the initial vaccination of cattle with a full dose of the vaccine from strain $B$. abortus 82 is fraught with post-vaccination abortions. In order to solve this problem, in the production conditions of one of

Corresponding Author: Alexei Novitsky

na.lescheva@omgau.org

Published: 5 April 2021

Publishing services provided by Knowledge E

(c) Alexei Novitsky et al. This article is distributed under the terms of the Creative Commons Attribution License, which permits unrestricted use and redistribution provided that the original author and source are credited.

Selection and Peer-review under the responsibility of the DonAgro Conference Committee. the farms where the current epizootic situation for brucellosis required alternative approaches for containment of a brucellosis outbreak, a small dose of the vaccine was tested on the cattle without specific immunity. This resulted in immunogenicity, loss of abortogenicity and the presence of provocative properties of the vaccine in small doses. This allowed the detection of antibodies in animals with a hidden infection in the early stages after vaccination. Serological methods (AT, RBT and IDR with O-polysaccharide antigen), which are generally accepted in veterinary practice, were used as diagnostic tests. The reimmunization of the cows with a small dose of the vaccine from strain 82 allowed diagnostic tests to be carried out earlier after vaccination than after the use of a full dose of the vaccine, which made it possible to diagnose brucellosis in a short period of time after vaccination. The provocation of immunological reactions in latently sick animals after the vaccination with small doses, and the isolation of those who responded under the condition of supported immunity made it possible to achieve the recovery of farm animals in a short period of time, which was proved by negative indicators of the immunodiffusion reaction with O-polysaccharide antigen.

Keywords: brucellosis, small doses of the vaccine, abortogenicity, immunity

\section{Introduction}

G OPEN ACCESS
The improvement of the system of anti-brucellosis measures is the ultimate goal of all studies conducted on the problem of brucellosis. Despite the impressive results 
achieved in the Russian Federation in relation to the elimination of this disease, there are some unresolved issues related to the cattle immune defense in case of the sudden brucellosis outbreaks in the brucellosis-free regions. Most often, such situations arise after the cessation of specific prophylaxis against brucellosis in the areas that are at risk of infection from the neighboring regions or states [1-3]. The system of specific prophylaxis against brucellosis, established in Russia, is based on the use of a vaccine from strain B. abortus 82 . The developed schemes for the use of this vaccine made it possible to eliminate its abortogenic properties due to residual immunity from primary vaccinations of calves at 4-5 months of age and heifers before insemination [2].

\section{Methods and Equipment}

Building up immunity in the breeding stock that has not been previously immunized is considered to be problematic. The previous practices show that the use of this vaccine in a full dose (100 billion m.c.) on incalvers intact with respect to brucella leads to massive post-vaccination abortions [4]. The non-abortogenic anti-brucellosis vaccine from strain 19 available in veterinary practice cannot be used on adult animals due to its high agglutinogenicity $[5,6]$. Many researchers propose the use of a vaccine from strain B. abortus 19 in a small dose (3 billion m.c.), which does not possess abortogenic properties being sufficiently immunogenic, and does not cause long-term production of post-vaccination antibodies, compared to the use of the vaccine in a full dose [7-14]

These studies prompted us to conduct experimental and production research works on the use of small doses of the vaccine from strain 82 in order to determine the period of time when post-vaccination reactions start to fade, and to solve other urgent issues related to the level of immunity formed, reduction of abortogenicity and epizootological effectiveness. In previous studies [1, 15], it was found out that reimmunization of cows with a small dose of the vaccine from strain 82 allows diagnostic tests to be carried out earlier after vaccination than with the full dose of the vaccine (Table 01).

According to Table 01, it is possible to do diagnostic tests one month after the revaccination performed in accordance with the schemes where small doses of the vaccine from strain 82 were used. At the same time, after vaccination with a full dose, serological tests could be carried out no earlier than two months after the vaccination.

The results of the experiment aimed to determine the intensity of immunity in cows, immunized with vaccines from strains 19 and 82 in different doses, are shown in Table 02. 
TABLE 1: The dynamics of the formation of post-vaccination antibodies detected by AT, CFT, RBT after reimmunization of cows with a vaccine from strain 82 in different doses

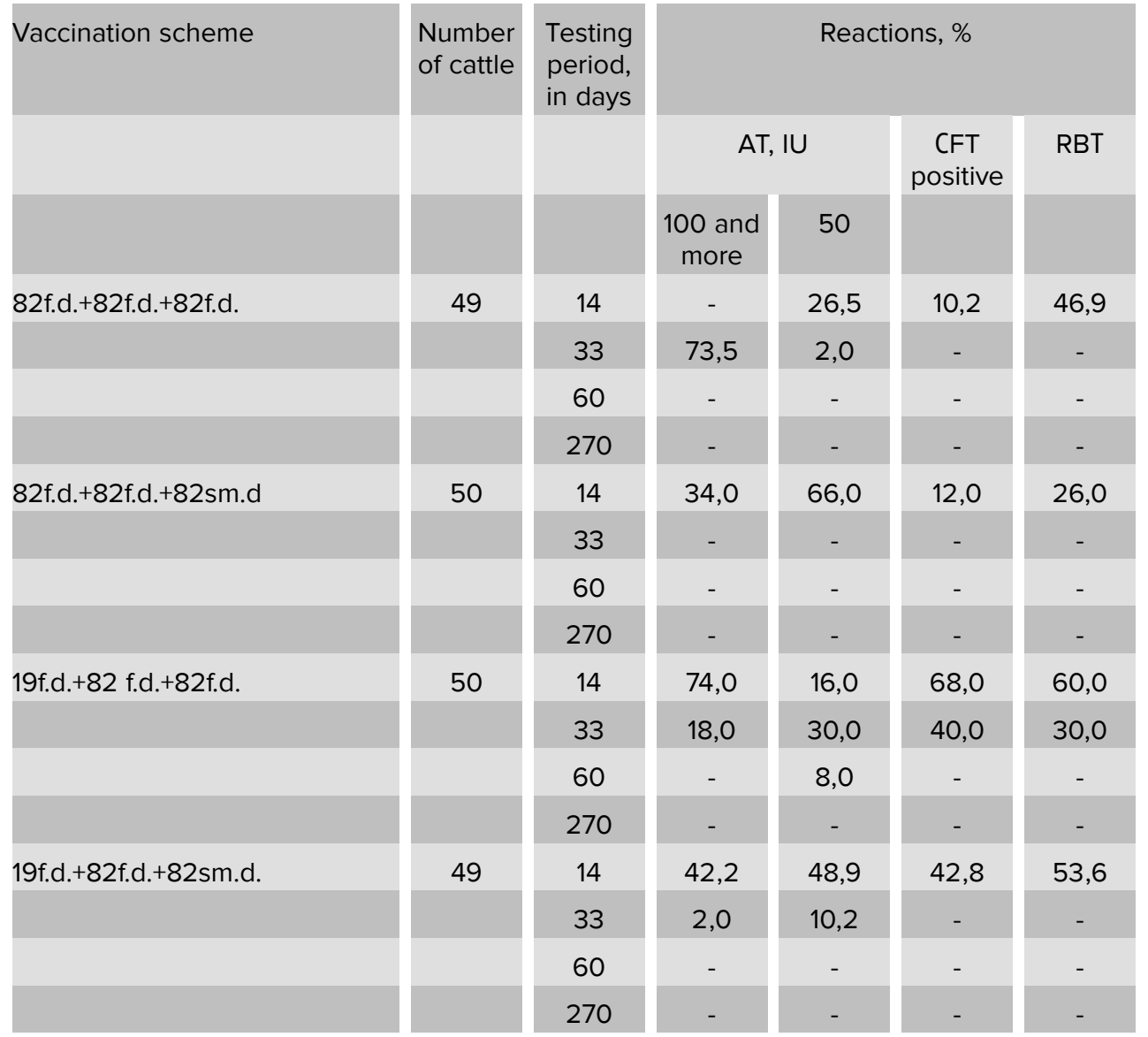

Note: f.d. - full dose of vaccine, sm.d. - small dose of vaccine, «-» - negative result

The data presented in Table 02 indicate that there is no significant difference in the immune resistance of animals vaccinated according to different schemes. Obviously, triple immunization, regardless of the vaccine and dose, provided approximately equal immune protection in $60 \%$ of cattle. It is generally accepted that $50-70 \%$ of the immunized cattle in the herd is a high indicator of immune protection.

The obtained experimental data from testing a small dose of the vaccine from strain 82 were the basis for further production experiments.

The production experiments on testing small doses of the vaccine were carried out on the farm in the North Kazakhstan region. Due to the fact that the farm is located in the area neighboring to the Omsk region, Omsk State Agrarian University was commissioned to provide scientific and veterinarian assistance in the recovery of the cattle from brucellosis.

Before the start of the production experiments on the use of small doses of the vaccine from strain 82 , an epizootic situation was monitored for cattle brucellosis on 
TABLE 2: The formation of immunity in cows vaccinated against brucellosis according to different schemes (vaccine strains 19 and 82)

\begin{tabular}{|c|c|c|c|c|c|c|}
\hline \multirow{2}{*}{$\begin{array}{l}\text { Vaccination scheme (vaccine } \\
\text { strain) }\end{array}$} & \multirow{2}{*}{$\begin{array}{l}\text { Number of } \\
\text { cattle }\end{array}$} & \multicolumn{3}{|c|}{ Infected } & \multirow{2}{*}{$\begin{array}{l}\text { Number of } \\
\text { immun. } \\
\text { cattle (\%) }\end{array}$} & \multirow{2}{*}{$\begin{array}{l}\text { Index of } \\
\text { infection }\end{array}$} \\
\hline & & $\mathrm{S}$ & $\mathrm{R}$ & Total & & \\
\hline 19 f.d. +19 f.d. +19 sm.d. & 5 & 2 & 0 & 2 & 60 & 10,4 \\
\hline 19 f.d. +19 sm.d. +19 sm.d. & 5 & 1 & 0 & 1 & 80 & 3,2 \\
\hline 19 f.d. +82 f.d. +82 f.d. & 5 & 1 & 1 & 2 & 60 & 2,4 \\
\hline 19 f.d. +82 f.d. +82 sm.d. & 5 & 1 & 1 & 2 & 60 & 2,4 \\
\hline 82 f.d. +82 f.d. +82 f.d. & 4 & 1 & 1 & 2 & 50 & 3,0 \\
\hline 82 f.d. +82 f.d. +82 sm.d. & 5 & 1 & 1 & 2 & 60 & 3,2 \\
\hline Control & 3 & 3 & 0 & 3 & 0 & 24,0 \\
\hline
\end{tabular}

Note: S - Systemic form of infection, R - Regional form of infection, f.d. - full dose of vaccine, sm.d. - small dose of vaccine

the farms of adjacent Omsk and North Kazakhstan regions. It was found out that there was a decrease in the vaccine load, and consequently in the level of immune defense in the cattle. While it can be justified in relation to the farms in the Omsk region due to their long brucellosis-free status, the refusal from the specific prophylaxis on the Kazakhstan farms, where there is a continuing threat of infection, was clearly premature.

The analysis showed that the current epizootic situation in relation to brucellosis on the farm required more advanced approaches to containment of a cattle brucellosis outbreak. It was due to the fact that the imported adult cattle were intact to brucellosis antigen, i.e. the animals lacked immunity to brucellosis, and the administration of a full dose of the reactogenic vaccine from strain 82 would only exacerbate the epizootic situation. In this regard, in order to avoid the negative consequences, it was decided to use this vaccine in a tenfold reduced dose.

The proposed plan of preventive measures included two main activities. The first one is to build up the immunity in adult animals avoiding post-vaccination abortions. The second one is to apply methods for identifying animals with a latent form of brucellosis in the early period after the vaccination. In order to avoid post-vaccination abortions, it was decided to simultaneously immunize the entire herd with a vaccine from strain 82 with a small dose. It allowed the containment of infection by creating an immune background and provocation of immune reactions identified by serological methods in latently sick animals

Serological methods (AT, RBT, IDR with O-polysaccharide antigen) generally accepted in veterinary practice were used as diagnostic tests. Long-term studies aimed to determine the diagnostic significance of the immunodiffusion reaction with O-polysaccharide 
antigen gave reason to recognize it as a method for assessing the epizootic situation about brucellosis in herds of vaccinated cattle $[16,17]$.

\section{Results and Discussion}

Confirming results were obtained on two brucellosis-free farms in a short period of time after immunization of cows with small doses of vaccine from strain 82 (Tables 03 and 04).

TABLE 3: Indicators of serological tests of brucellosis-free cattle immunized with a small dose of the vaccine from strain 82 (“Zeleny Gaj” Farm)

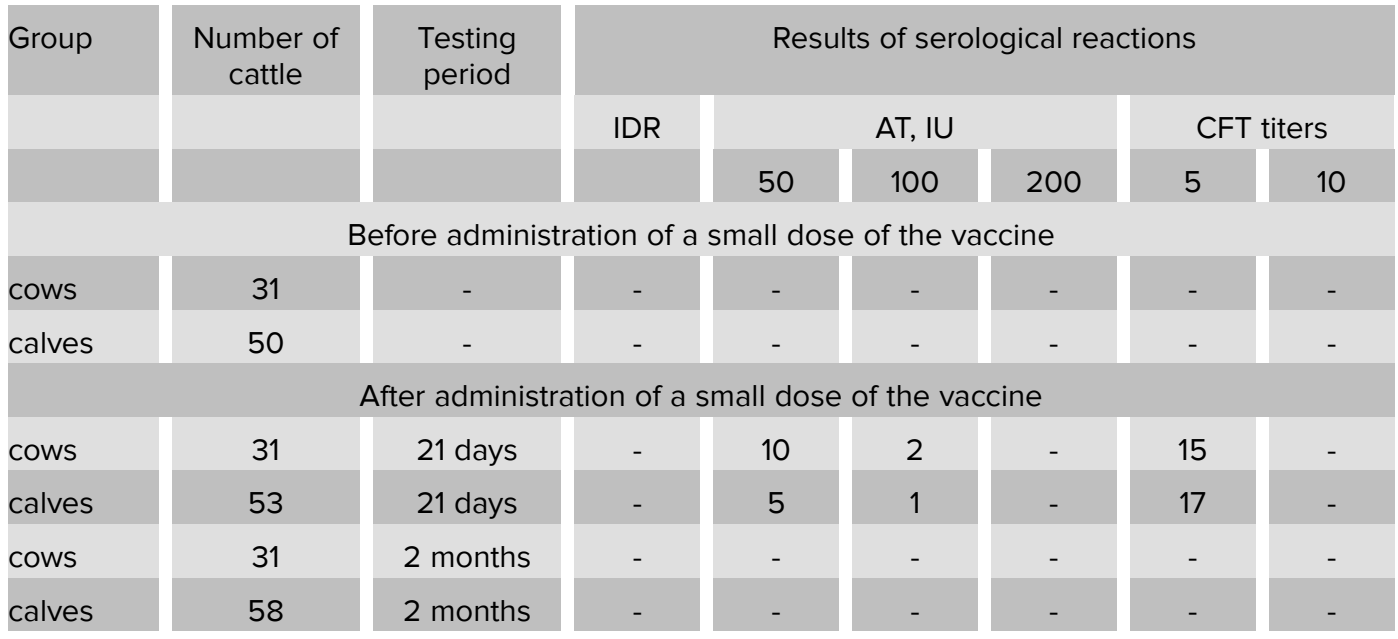

Note: «-»- negative result. The brucellosis-reacting cattle were not isolated from the herd.

TABLE 4: Indicators of the immunodiffusion reaction with O-polysaccharide antigen after immunization of cows on a brucellosis-free farm with a small dose of vaccine from strain 82 ("Tsentralnaya" Farm).

Period of time after
immunization
21 days
1,5 month
3,5 months

$\begin{gathered}\text { Number of } \\ \text { tests }\end{gathered}$
239
145
140

\begin{tabular}{|c|c|c|c|}
\hline \multicolumn{3}{|c|}{ Results (\%) } \\
\hline \multicolumn{2}{|c|}{ AT, IU } & \multicolumn{3}{c|}{ CFT } \\
\hline 50 & 100 & 5 & 10 \\
\hline 58,0 & - & 41,0 & - \\
\hline 42,3 & 2,7 & 10,3 & 6,2 \\
\hline 29,4 & - & 11,4 & 0,7 \\
\hline
\end{tabular}

IDR Positive

Note: «-» - negative result. The brucellosis-reacting cattle were not isolated from the herd

It can be seen from the tables that in the short period of time after immunization, small doses of the vaccine induced the synthesis of agglutinins and complement-binding antibodies mainly in low titers with a tendency to fade. The negative indicators of the immunodiffusion reaction proved the fact that the herd was free from brucellosis.

Other results of serological tests on brucellosis were received from the cattle on the infected farm (Table 05). 
The data, shown in Table 05, indicate that at the time of administration of small doses of the vaccine to the herd on the "Kumysnoe" farm, some cattle were infected with brucellosis. Before the first vaccination, positive indicators of immunodiffusion reaction (IDR) and AT, CFT in high titers were identified in the herd. After the isolation of the brucellosis-reacting cows, the rest were vaccinated with a small dose of vaccine from strain 82 in order to stop the outbreak and to provoke reactions in animals with a latent course of infection. The vaccinated cattle were tested with the use of AT, CFT and IDR 21 days, 2 and 3 months after the vaccination. 3 months later after the isolation of animals reacting in high titers detected by AT (200 IU), CFT (1:20) and IDR, the rest cattle were revaccinated with a small dose of the vaccine and 2 months later, the obtained results indicated recovery, confirmed by negative IDR indicators.

TABLE 5: Serological indicators in cows on the infected farm (“Kumysnoe” Farm)

\begin{tabular}{|c|c|c|c|c|c|c|c|c|}
\hline \multirow{3}{*}{$\begin{array}{l}\text { Number of } \\
\text { cattle }\end{array}$} & \multirow[t]{3}{*}{ Testing period } & \multicolumn{7}{|c|}{ Results of serological reactions } \\
\hline & & \multirow[t]{2}{*}{ IDR } & \multicolumn{3}{|c|}{ AT, IU } & \multicolumn{3}{|c|}{ CFT titers } \\
\hline & & & 50 & 100 & 200 & 5 & 10 & 20 \\
\hline 206 & $\begin{array}{c}\text { Not } \\
\text { vaccinated }\end{array}$ & 6 & 18 & 4 & 4 & 12 & 6 & 5 \\
\hline \multicolumn{9}{|c|}{ Vaccination with a small dose of the vaccine from strain 82} \\
\hline 195 & 21 days & - & 27 & 79 & 10 & 21 & 29 & 32 \\
\hline 174 & 2 months & 1 & 24 & 18 & 3 & 27 & 16 & 1 \\
\hline 168 & 3 months & - & 5 & 2 & - & - & - & - \\
\hline \multicolumn{9}{|c|}{ Revaccination with a small dose of the vaccine from strain 82} \\
\hline 154 & 21 days & 4 & 31 & 28 & 3 & 23 & 19 & 7 \\
\hline 158 & 2 months & - & 10 & 8 & 2 & 5 & 2 & - \\
\hline
\end{tabular}

Note: After every test, the brucellosis-reacting cattle were isolated from the herd. «-» - negative result.

TABLE 6: Dynamics of post-vaccination reactions in cows in a recovering herd after administration of a small dose of the vaccine from strain 82 ("Cherny Stan" farm)

\begin{tabular}{|c|c|c|c|c|c|c|c|c|c|c|c|}
\hline \multirow{3}{*}{$\begin{array}{l}\text { Testing } \\
\text { period }\end{array}$} & \multirow{3}{*}{$\begin{array}{l}\text { Number } \\
\text { of tests }\end{array}$} & \multicolumn{10}{|c|}{ Results } \\
\hline & & \multicolumn{4}{|c|}{ AT, IU } & \multicolumn{4}{|c|}{ CFT } & \multirow[t]{2}{*}{ RBT } & \multirow[t]{2}{*}{ IDR } \\
\hline & & 50 & 100 & 200 & 400 & 5 & 10 & 20 & 40 & & \\
\hline \multicolumn{12}{|c|}{ Before vaccination with a small dose of the vaccine } \\
\hline & 312 & 30 & 8 & 6 & 8 & 17 & 8 & 6 & 14 & 56 & 42 \\
\hline \multicolumn{12}{|c|}{ After vaccination with a small dose of the vaccine } \\
\hline 1 month & 330 & 200 & 49 & - & - & 71 & 18 & 23 & 18 & 225 & 11 \\
\hline 3 months & 239 & 41 & 2 & 3 & 1 & 1 & 0 & 2 & 0 & 54 & 5 \\
\hline 5 months & 202 & 18 & 6 & 4 & 1 & 3 & 7 & 5 & 0 & 10 & 1 \\
\hline
\end{tabular}

Note: The cattle with titers detected by AT 200-400 IU and CFT 1:20-1:40 were isolated from the herd. 
Significantly, the immunodiffusion reaction with O-polysaccharide antigen was detected in the period of 1-3 months (11 animals had 3.3\% 1 month later; 5 animals had $2.0 \% 3$ months later; 1 animal had $0.4 \% 5$ months later) with the subsequent fading of indicators of all serological reactions. Further, the cows in the herd were reimmunized with a full dose of the vaccine from strain 82 and heifers vaccinated with the same vaccine were added to the herd. This herd was under veterinarian supervision; manifestations of positive reactions were not noted.

The important result was that the use of a small dose of the vaccine from strain 82 did not lead to post-vaccination abortions in heifers in the herds

\section{Conclusion}

The long-term brucellosis-free cattle farms in the regions of the Russian Federation serve as justification for a reduction of the cattle immunization programmes with a further complete refusal from the specific prophylaxis against this disease. Nevertheless, such measures can only be implemented in case of a full guarantee that the infection will not be introduced in brucellosis-free territories such as the areas of the central Russia and other adjacent regions.

Unfortunately, according to statistics, in some regions of Russia, there are infected areas where new cattle brucellosis outbreaks occur. The main reason is the noncompliance with the provisions of the system of special anti-brucellosis measures and, first of all, the non-fulfillment of regulations for specific prophylaxis with an existing threat of infection. The premature removal of restrictions and cessation of immunization procedures resulted in the accumulation of non-immune animals in herds, which led to the cattle brucellosis outbreaks. The attempts to contain the outbreaks of infection by systematic testing and isolation of the brucellosis-reacting cattle did not bring desired results, since the infection of non-immune cattle was ahead of its detection. In such cases, the spread of the disease can be stopped only by simultaneous immunization of the entire livestock. However, vaccination of adult non-immune animals with different periods of pregnancy carries an even greater danger associated with massive postvaccination abortions, exacerbating the course of brucellosis.

Based on the foregoing, the use of small doses of the vaccine from strain 82 in the vaccination scheme against brucellosis allows solving a number of problems. Firstly, it is possible to weaken the reactogenic properties of the vaccine and thereby avoid post-vaccination abortions during immunization of pregnant animals in cases of urgent need. Secondly, it is possible to maintain the immune status of the adult cattle without 
post-vaccination complications. Thirdly, provocative properties of the vaccine make it possible to identify animals with a latent form of brucellosis in the early stages after vaccination, which shortens the recovery period for the herd.

\section{Conflict of Interest}

The authors declare that there is no conflict of interest.

\section{References}

[1] Maskaev, N. N., et al. (1988) Immunological Reactions and Immunity in Cattle Vaccinated with Anti-Brucellosis Vaccines in Different Doses. In Methods of Diagnosis and Prevention of Animal Brucellosis and Tuberculosis: Research Materials of VNIIBTZH. (pp. 26-31) Omsk: SO VASKHNIL

[2] Novitsky, A. A. (2001). Omsk Region as a Model for Creating a System of AntiBrucellosis Measures. Infectious Animal Pathology: Research Materials of SO RASKHN. Omsk: VNIIBTZH.

[3] Novitsky, A. A., et al. (2019). Areas of Concern of Brucellosis Specific Prevention. Advances in Animal and Veterinary Sciences, vol. 7, pp. 94-99.

[4] Kosilov, I. A. (1985). The Role of Specific Prevention in the System of Anti-Brucellosis Measures. In Epizootol. and Infection Control Measures against Animal Diseases: Research Materials. Novosibirsk: SO VASKHNIL.

[5] Salmakov, K. M. (1970). Testing a Vaccine from a Slightly Agglutinogenic Strain of Brucella 82 In Cattle. Research Notes of Kazan Vet. Inst., vol. 107, pp. 83-87.

[6] Yudina, E. and Konovalov, S. (2019). Development Issues and Prospects of Milk Processing Enterprises. Presented at The Fifth Technological Order: Prospects for the Development and Modernization of the Russian Agro-Industrial Sector" (TFTS 2019): Vol. 393). October 15th, 2019 (Omsk, Russia). Electronic Materials (pp 436-439) Paris: Atlantis Press.

[7] Albertyan, M. P. (1990). Immunogenicity of Vaccines from Strains 19, 104 M and 82 for Cattle in Various Schemes of Application. Bulletin VIEV, vol. 73. pp. 97-103.

[8] Grinko, V. K., et al. (1987). Study of the Effectiveness of Immunization of Cattle with a Vaccine from Strain 19 in a Small Dose. Actual problems of veterinary science and practice: Abstr. of Scient Conf. March 26-27th, 1987 (Samarkand, Uzbek SSR). (pp. 87) Samarkand: SO VASKHNIL. 
[9] Dimova, A. S. (2003). Efficiency of Various Anti-Brucellosis Vaccines and Schemes for their Use in Cattle and Goats. (Dissertation of the candidate of veterinary sciences dissertation, Novosibirsk: IEVSDV).

[10] Kim, V. I. (1989). The Results of Testing the Vaccine from Strain 19 in a Small Dose in the Kyrgyz SSR. In Abstracts of the All-Union Conference. Moscow: Znanie Novosibirsk.

[11] Minzhasov, K. I. (1986). The Effectiveness of Vaccination of Cattle with a Vaccine from Strain 19 in a Small Dose. In K. I. Minzhasov, (Ed.), Problems of Intensification of Animal Husbandry in Kazakhstan. (pp. 75-76) Alma-Ata: Kazakh republican government scientific and technical society of agriculture.

[12] Romakhov, V. A., et al. (1989). Epizootological Efficacy of a Vaccine from Strain 19 in a Small Dose against Cattle Brucellosis on Farms in the Samarkand region of the Uzbek SSR. Abstracts of the All-Union Conference. Moscow: Znanie Novosibirsk.

[13] Rusakov, Y. V., et al. (1989). Immunity against Brucellosis in Cattle Reimmunized with Small Doses of Agglutinogenic Vaccines. Actual Problems of Tuberculosis and Brucellosis in Agricultural Animals: Research Materials of VASKHNIL. Novosibirsk: Sib. Department.

[14] Khlystunov, A. G., et al. (1988). Immunological Reactivity of Heifers after Revaccination with Small Doses of the Vaccine from Strains 19 and 104 M. Novosibirsk: VASKHNIL, IEVSDV.

[15] Krasikov, A., et al. (2019). Dynamics of Immunological Parameters of Cattle Infected with Leukemia Virus and its Correction with Betulin-Based Medication. The Fifth Technological Order: Prospects for the Development and Modernization of the Russian Agro-Industrial Sector (TFTS 2019): Vol. 393. October 15th, 2019 (Omsk, Russia). Electronic Materials (pp 25-28) Paris: Atlantis Press. Striełkowski).

[16] Diaz, R. (1979). Radial Immunodifusion Test with a Brucella Polysaccharide Antigen for Differentiating Infected from Vaccinated. Journal of Clinical Microbiology, vol. 10, pp. 37-41.

[17] Cherwonogrodzky, J. W. (1988). Brucella abortus 1119-3 O-chain Polysaccharide to Differentiate Sera from B. abortus S-19 - Vaccinated and Fieldstrain Infected Cattle by Agar Gel Immunodiffusion. Journal of Clinical Microbiology., vol. 26, issue 6, pp. $1120-1123$. 